\title{
AC 2010-235: STANDING AND TRAVELING WAVES ON TRANSMISSION LINES:GETTING IT RIGHT
}

\section{Raymond Jacquot, University of Wyoming}

Ray Jacquot received his BSME and MSME degrees at the University of Wyoming in 1960 and 1962 respectively. He was an NSF Science Faculty Fellow at Purdue University where he received the Ph.D. in 1969. He joined the Electrical Engineering faculty of the University of Wyoming in 1969. He is a Fellow of ASEE, a Life Senior Member of IEEE and Life Member of ASME. He has been active in ASEE for over four decades serving as Rocky Mountain Section Chair and PIC IV Chair. His professional interests are in modeling, control and simulation of dynamic systems. He is currently Professor Emeritus of Electrical and Computer Engineering. E-mail: quot@uwyo.edu.

\section{David Voltmer, Rose-Hulman Institute}

David R. Voltmer received degrees from Iowa State University (BSEE), University of Southern California (MSEE), and The Ohio State University (PhD EE). During nearly four decades of teaching, Dr. Voltmer has maintained a technical focus in electromagnetics, microwaves, and antennas. His more recent efforts are directed toward the design process and project courses. He has served in many offices of the ERM division of ASEE and in FIE. Dr. Voltmer is an ASEE Fellow and a Life Senior member of IEEE. E-mail: voltmer@rose-hulman.edu

\section{John Steadman, University of South Alabama}

John Steadman received degrees from the University of Wyoming (BSEE and MSEE) in 1964 and 1966 respectively. After graduation he was employed by General Dynamics. He received the PhD EE from Colorado State University in 1971 prior to joining the faculty of Electrical Engineering at the University of Wyoming in 1971 where he held all ranks and served as Department Head and Associate Dean. Dr. Steadman served the profession as President of the National Council of Examiners for Engineers and Surveyors and as President of IEEE-USA. He has been active in ASEE for over 3 decades serving as Section Chair and Division Treasurer and is an ASEE Fellow. His professional interests are in bioengineering, digital systems and real-time signal processing. Dr Steadman is currently Dean of Engineering at the University of South Alabama. E-mail: jsteadman@usouthal.edu 


\section{Standing and Traveling Waves on Transmission Lines: Getting it Right}

\section{Introduction}

In the recent engineering education literature there have been numerous papers dealing with strategies for teaching electrical transmission lines. These papers approach the assistance in teaching from two points of view. The first is the use of simple low-cost experiments to demonstrate the concepts ${ }^{1-3}$ and the second approach employs computer animation ${ }^{4,5}$. Another recent paper discusses the concept of teaching transmission lines early in the electromagnetics sequence ${ }^{6}$.

Seeking clarification to the problem of sinusoidally driven, arbitrarily terminated, lossless transmission lines one of the authors asked the question, "How does the voltage on the line behave?" In order to answer this question some MATLAB ${ }^{\mathrm{TM}}$ scripts were written to animate the voltage on the line. In examining these animations more questions arose about the concept of standing waves. In the examination of the animations the authors were confounded because for arbitrary termination impedance the resulting waveform did not "stand" as is the case for the commonly discussed short-circuit and open-circuit terminations. Consultation of a number of electromagnetics textbooks found that the term "standing wave" is often used to describe the interference pattern created by a combination of forward and backward traveling waves of the same wavelength (or frequency). As we shall show this definition is incomplete and leads to a semantic error in a number of contemporary textbooks.

\section{Standing or Stationary Waves}

After the search of electromagnetics textbooks it was decided to look on the web for information which is contained on physics websites. Standing waves are created by the addition of two oppositely traveling waves of equal wavelength and amplitude. There seems to be an agreement that the terms standing waves and stationary waves are synonymous and that such waves are characterized by nodes (points of zero response) and antinodes (points of maximum response) and that there is no energy transport in the direction of propagation ${ }^{7}$.

A final resolution to the definition problem came with a search of Lord Rayleigh's 1891 edition of The Theory of Sound ${ }^{8}$ wherein the above stated definition was confirmed. The Rayleigh definition essentially states that sinusoidal waves are stationary (standing) if they are of the form

$$
w(z, t)=P \cos (\omega t-\varepsilon) \cos (\beta z-\alpha)
$$

or after employing the appropriate trigonometric identity

$$
w(z, t)=\frac{P}{2}[\cos (\omega t+\beta z-\varepsilon-\alpha)+\cos (\omega t-\beta z-\varepsilon+\alpha)]
$$

We see that expression (2) is the sum of forward and backward traveling waves each of amplitude P/2. A similar result may be found in the handbook of Korn and Korn ${ }^{9}$. 


\section{Some Transmission Line Theory}

Consider the lossless transmission line illustrated in Figure 1 with source impedance $Z_{g}$ and terminal impedance $Z_{L}$.

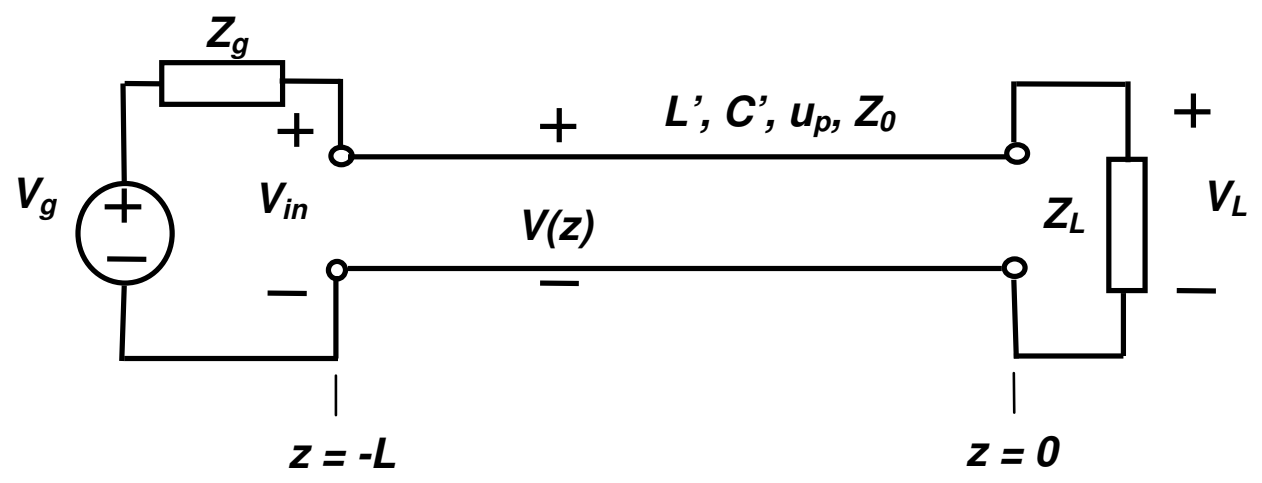

Figure 1. Lossless Transmission Driven by a Sinusoidal Source.

The steady-state sinusoidal voltage and current on the line are governed by the phasor form of the telegrapher's equations or

$$
\frac{d V}{d z}=-j \omega L^{\prime} I
$$

and

$$
\frac{d I}{d z}=-j \omega C^{\prime} V
$$

where $L^{\prime}$ and $C^{\prime}$ are the respective per unit length inductance and capacitance and $V(z)$ and $I(z)$ are the phasor voltage and current as functions of location. The characteristic impedance of the line is $Z_{0}=\sqrt{L^{\prime} / C^{\prime}}$ and the propagation velocity $u_{p}=1 / \sqrt{L^{\prime} C^{\prime}}$.

The solution for the phasor voltage on the line after application of the terminal boundary condition is

$$
V(z)=V_{0}^{+}\left[e^{-j \beta z}+\Gamma e^{j \beta z}\right]
$$

where $\beta=\omega \sqrt{L^{\prime} C^{\prime}}$ and $\Gamma$ is the complex reflection coefficient defined by

$$
\Gamma=\frac{Z_{L}-Z_{0}}{Z_{L}+Z_{0}}=|\Gamma| e^{j 2 \theta}
$$

In some of the engineering education literature the interference pattern given by calculation of the magnitude of (5) for any nonzero value of $\Gamma$ is termed a standing wave. As will now be shown this solution of expression (5) does not, in general, result in a standing wave. Using the polar form for $\Gamma, V(z)$ may be written as

$$
V(z)=V_{0}^{+} e^{j \theta}\left[e^{-j(\beta z+\theta)}+|\Gamma| e^{j(\beta z+\theta)}\right]
$$

For any general termination expression (5) may be rewritten as

$$
V(z)=V_{0}^{+} e^{j \theta}\left\{|\Gamma|\left[e^{-j(\beta z+\theta)}+e^{j(\beta z+\theta)}\right]+(1-|\Gamma|) e^{-j(\beta z+\theta)}\right\}
$$

In general $V_{0}^{+}$is complex and can be written as $\left|V_{0}^{+}\right| e^{j \phi}$ so $V(z)$ is 


$$
V(z)=\left|V_{0}^{+}\right| e^{j(\phi+\theta)}\left\{|\Gamma|\left[e^{-j(\beta z+\theta)}+e^{j(\beta z+\theta)}\right]+(1-|\Gamma|) e^{-j(\beta z+\theta)}\right\}
$$

and the resulting time domain solution is

$$
v(z, t)=\left|V_{0}^{+}\right|\{2|\Gamma| \cos (\beta z+\theta) \cos (\omega t+\phi+\theta)+(1-|\Gamma|) \cos (\omega t-\beta z+\phi)\}
$$

The first term is a standing wave as defined by Rayleigh in expression (1) and the second term a forward traveling wave and clearly the general solution to this problem is not simply a standing wave as purported in some of the textbook literature but rather a superposition of both a standing wave and a forward traveling wave. It appears that this semantic error has been in the textbook literature for at least half a century or perhaps much longer.

If $|\Gamma|=1$ then relation (10) becomes

$$
v(z, t)=2\left|V_{0}^{+}\right| \cos (\beta z+\theta) \cos (\omega t+\phi+\theta)
$$

which is a standing wave as defined by Rayleigh. ${ }^{8}$ From relation (6) it is easy to see that the only way for $|\Gamma|=1$ is for $Z_{L}$ to be zero, infinity or imaginary (reactive).

If $|\Gamma|=0$ then the solution becomes

$$
v(z, t)=\left|V_{0}^{+}\right| \cos (\omega t-\beta z+\phi)
$$

which is a forward traveling wave. An expression similar to (10) for the current on the line can be developed and then both can be evaluated at $z=0$ and then the average power at the load may be evaluated and we can see that this power is transmitted to the load by only the forward traveling wave portions of the voltage and current expressions.

There are several misnomers that persist as a result of this semantic problem. The first is the term the standing wave diagram which is given by plotting the magnitude of either relation (5) or (9) as a function of $z$ regardless of the value of $\Gamma$. This might better be referred to as the amplitude diagram since it represents the voltage amplitude along the line. The standing wave $\underline{\text { ratio }}$ is the other misnomer and is the ratio of the maximum to the minimum of the so called standing wave diagram.

\section{An Example}

Consider the lossless line with the following parameter values

$$
\begin{array}{ll}
Z_{0}=300 \Omega & L=5200 \mathrm{~m} \\
Z_{g}=300 \Omega & u_{p}=2 \times 10^{8} \mathrm{~m} / \mathrm{s} \\
f=10^{5} \mathrm{~Hz} & V_{g}=1\left\llcorner 0^{\circ} \mathrm{V}\right.
\end{array}
$$

We shall examine what happens as we vary the load impedance $Z_{L}$ by considering three cases:

1) $Z_{L}=\infty$ (open circuit termination, $|\Gamma|=1$ ), 2) $Z_{L}=300 \Omega$ (the matched case, $|\Gamma|=0$ ) and 3) $Z_{L}$ $=500 \Omega$ (the arbitrary unmatched case, $|\Gamma|=0.25$ ).

Case $1 Z_{L}=\infty$ (Open Circuit Termination)

The voltage on the line is shown in Figure 2 for 10 values of time over one temporal period. In this case the voltage on the line is a standing wave with the associated nodes and antinodes. 


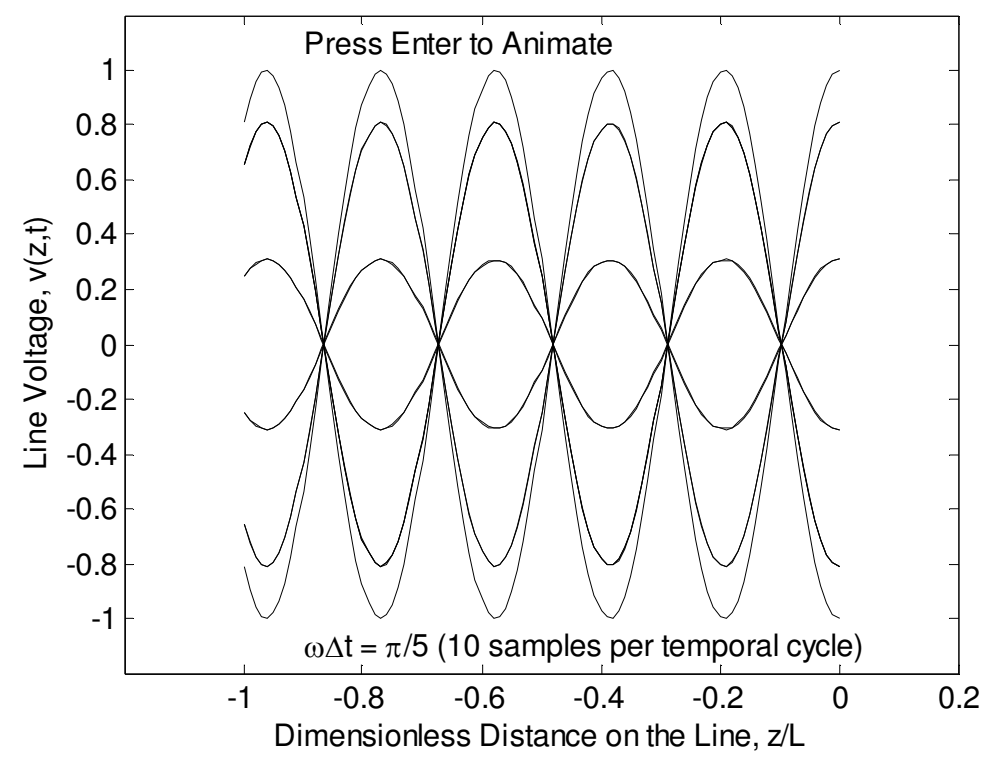

Figure 2. Standing Waves on Transmission Line with Open Circuit Termination, $Z_{0}=300 \Omega, Z_{L}=\infty \Omega$.

\section{Case $2 Z_{L}=300 \Omega$ (Matched Termination)}

Figure 3 illustrates the line voltage for the case where the terminal impedance is matched to the characteristic impedance of the line $(300 \Omega)$. This is a forward traveling wave with an amplitude of 0.5 volts

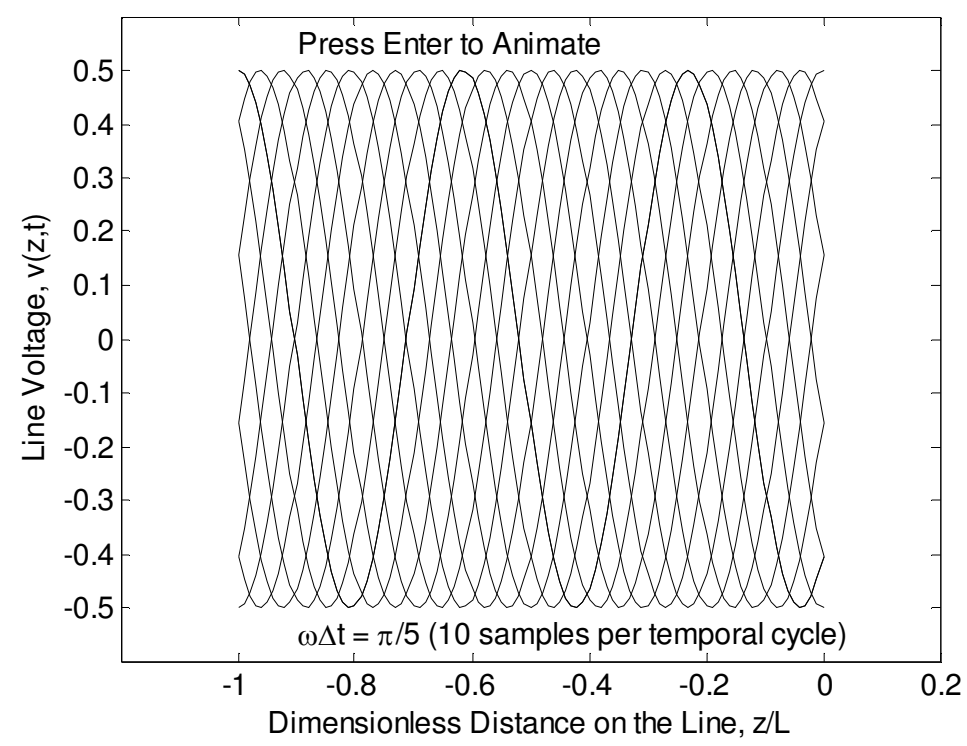

Figure 3. Traveling Voltage Waves for a Terminal Impedance Matched to the Line, $Z_{0}=Z_{L}=300 \Omega$. 


\section{Case $3 Z_{L}=500 \Omega$ (Arbitrary Unmatched Termination)}

Figure 4 illustrates the voltage on the line for a mismatched load impedance. The traveling wave nature can be seen clearly superimposed over the standing wave. There are no nodes in this case as opposed to those shown in Figure 2. The solution is neither a pure standing wave nor a pure traveling wave but a combination of the two.

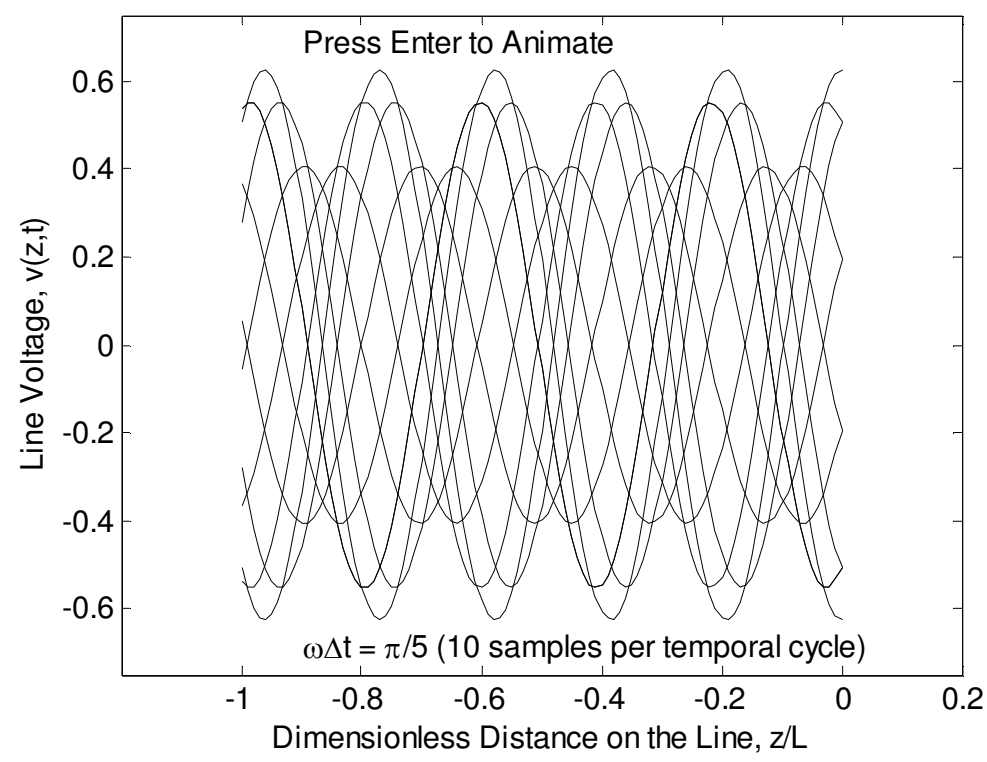

Figure 4. Forward Traveling and Standing Voltage Wave for an Unmatched Termination, $Z_{0}=300 \Omega, Z_{L}=500 \Omega$.

For the case of a short circuit termination or a reactive termination the magnitude of the reflection coefficient is unity and the voltage waveform on the line is similar to that of Figure 2.

\section{Conclusion}

The authors have shown that the only when a lossless transmission line is terminated by a load without energy dissipation can standing waves alone develop on the line. In any other case the waves on the line are a combination of a standing and a forward traveling wave.

In the process of exploring animation of the transmission line problem the first author became interested in how animation might clarify the teaching of other concepts described by partial differential equations. As a result more than fifty scripts were developed to animate problems in heat conduction, beam and string vibration, groundwater transport, wave propagation and fluid dynamics. The interested reader can access all these MATLAB ${ }^{\mathrm{TM}}$ scripts for animation at the website http://www.eng.uwyo.edu/classes/matlabanimate

\section{References}

1. Jalali, Transmission Line Experiments at Low Cost, Proc. 1998 ASEE Annual Conference and Exposition, Seattle, WA, June 28-July 1, 1998, Session 2648. 
2. D.M. Hata, A Low-Cost Approach to Teaching Transmission Line Fundamentals and Impedance Matching, Proc. 2004 ASEE Annual Conference and Exposition, Salt Lake City, UT, June 20-23, 2004, Session 1526.

3. A. Rusek and B. Oakley, Easy-to-do Transmission Line Demonstrations of Sinusoidal Standing Waves and Transient Pulse Reflections, Proc. 2007 ASEE Annual Conference and Exposition, Honolulu, HI, June 24-27, 2007, Session 3532.

4. C.W. Trueman, Teaching Transmission Line Transients Using Computer Animation, Proc. ASEE/IEEE Frontiers in Education Conference, San Juan, Puerto Rico, Nov. 10-13, 1999, Session 12a9.

5. R. G. Jacquot, C.H.G. Wright and R.F. Kubichek, Animation Software for Teaching Electrical Transmission Lines, Proc. 2006 ASEE Annual Conference and Exposition, Chicago IL, June 18-21, 2006, Session 1120.

6. S.H. Mousavinezhad, Electric and Magnetic Fields, Transmission Lines First?, Proc.2006 ASEE Annual Conference and Exposition, Chicago, IL, June 18-21, 2006, Session 3232.

7. www.citycollege.com/standing_wavesXIa.htm

8. J.W. Strutt, Third Baron Rayleigh, The Theory of Sound: Volume 1, Dover, New York, NY,1945.

9. G.A. Korn and T. M. Korn, Mathematical Handbook for Scientists and Engineers: Second Edition, McGraw-Hill, 1968. 